\title{
Brain pericytes increase the lipopolysaccharide-enhanced transcytosis of HIV-1 free virus across the in vitro blood-brain barrier: evidence for cytokine-mediated pericyte-endothelial cell crosstalk
}

Shinya Dohgu ${ }^{1}$ and William A Banks ${ }^{1,2^{*}}$

\begin{abstract}
Background: Human immunodeficiency virus-1 (HIV-1) enters the brain by crossing the blood-brain barrier (BBB) as both free virus and within infected immune cells. Previous work showed that activation of the innate immune system with lipopolysaccharide (LPS) enhances free virus transport both in vivo and across monolayer monocultures of brain microvascular endothelial cells (BMECs) in vitro.

Methods: Here, we used monocultures and co-cultures of brain pericytes and brain endothelial cells to examine the crosstalk between these cell types in mediating the LPS-enhanced permeation of radioactively-labeled HIV-1 (I-HIV) across BMEC monolayers.

Results: We found that brain pericytes when co-cultured with BMEC monolayers magnified the LPS-enhanced transport of I-HIV without altering transendothelial electrical resistance, indicating that pericytes affected the transcytotic component of HIV-1 permeation. As LPS crosses the BBB poorly if at all, and since pericytes are on the abluminal side of the BBB, we postulated that luminal LPS acts indirectly on pericytes through abluminal secretions from BMECs. Consistent with this, we found that the pattern of secretion of cytokines by pericytes directly exposed to LPS was different than when the pericytes were exposed to the abluminal fluid from LPS-treated BMEC monolayers.

Conclusion: These results are evidence for a cellular crosstalk in which LPS acts at the luminal surface of the brain endothelial cell, inducing abluminal secretions that stimulate pericytes to release substances that enhance the permeability of the BMEC monolayer to HIV.
\end{abstract}

Keywords: Blood-brain barrier, HIV-1, Pericytes, Brain endothelial cells, Cytokines, BMEC, AIDS, Neuroinflammation, Neuroimmune, Lipopolysaccharide

\footnotetext{
*Correspondence: wabanks1@uw.edu

'Department of Pharmaceutical Care and Health Sciences, Faculty of

Pharmaceutical Sciences, Fukuoka University, Fukuoka, Japan

${ }^{2}$ Geriatrics Research Education and Clinical Center, Puget Sound Health Care

System, Seattle WA and Division of Gerontology and Geriatric Medicine,

Department of Medicine, University of Washington, Bldg 1/Rm 810A,

VAPSHCS, 1660 S. Columbian Way, Seattle, WA 98108, USA
} 


\section{Background}

Human immunodeficiency virus-1 (HIV-1) enters the central nervous system (CNS) where it is relatively protected from anti-viral drugs [1-3]. Once in the CNS, HIV-1 has a number of neurotoxic effects that result in cognitive, hormonal, vascular, and homeostatic deficits [4-11]. HIV-1 in the CNS may also act as a source for re-infecting the periphery [12]. For these reasons, it is important to understand the mechanisms by which HIV-1 gains entry into the CNS.

HIV-1 must cross the blood-brain barrier (BBB) to enter the CNS. HIV-1 crosses the BBB both within infected immune cells by the Trojan horse mechanism $[13,14]$ and also as free virus [15]. These mechanisms are not passive but are highly regulated by intracellular machinery. Crossing within infected immune cells involves a form of diapedesis and is STAT-1 and JNKdependent $[16,17]$. In contrast, free virus entry is a multi-step transcytotic process $[15,18,19]$ that is JNKindependent but does depend on p38 mitogen-activated protein kinase [20] and glycoprotein interactions [21] with the mannose-6-phosphate receptor [22]. Both of these mechanisms, diapedesis of infected immune cells and free virus transcytosis, are enhanced by inflammation. This is relevant as HIV-1 and its viral proteins gp120 and TAT can activate the immune system and the cells that form the BBB [23-25]. It is important therefore, to further study the mechanisms by which neuroinflammation can enhance viral permeability across the BBB.

Lipopolysaccharide (LPS) application offers a useful model for studying the effects of neuroimmune activation on HIV-1 transport across the BBB. LPS is derived from the bacterial cell wall of gram negative bacteria [26] and is found in the circulation of HIV-1 patients as a result of gastroenteropathy-related bacterial translocation [27]. LPS enhances both immune cell [14] and free virus [20] transport across the BBB. LPS induces cytokine release into the blood and an even more prolonged immune response within the CNS [28]. LPS has many effects on the BBB and its cellular components [29], including altering paracellular and transcytotic permeability, altering brain-to-blood and blood-to-brain transporters [30-35], and inducing cytokine release [36,37], actions similar to those induced by HIV-1 and its proteins [38-43]. LPS should also prove useful for studying the cells that influence and modulate the BBB that together with brain endothelial cells form the neurovascular unit.

The neurovascular unit consists of endothelial cells, astrocytes, microglia, and other cells which are in constant crosstalk to inform one another of CNS events and to regulate $\mathrm{BBB}$ function [44]. Cytokines and other immunoactive substances are important messengers in this crosstalk. The least studied of these cells is the pericyte [45] that is also the cell in closest proximity to brain endothelial cells. The pericyte is a pluripotent cell and lies under the basement membrane surrounding CNS capillaries [46-48] and is known to be in intimate crosstalk with other cells of the neurovascular unit such as the astrocyte [49]. The pericyte is in direct contact with brain endothelial cells through both peg and socket joints and gap junctions. Recently, the pericyte has been shown to take up the HIV-1 virus and to be a site of viral replication [50].

Here, we examined the influence of pericytes on free virus transport across monolayers of brain microvascular endothelial cells (BMEC) and crosstalk between BMEC and pericytes in two experimental paradigms. First, we determined whether co-culture of BMEC with pericytes would alter the ability of free HIV-1 to cross the BMEC monolayer under both basal and LPS-treated conditions. Second, we examined the ability of pericytes to secrete cytokines either constitutively or induced by abluminal secretions from BMECs exposed to LPS. Together, the results show that LPS stimulates a crosstalk between brain endothelial cells and pericytes that enhance permeation of HIV-1 across BMEC monolayers.

\section{Methods}

\section{Radioactive labeling}

HIV-1 (MN) CL4/CEMX174 (T1) prepared and rendered non-infective by aldrithiol-2 treatment as previously described [51,52] was a kind gift of the National Cancer Institute, NIH. The virus was radioactively labeled by the chloramine-T method, a method which preserves vial coat glycoprotein activity $[53,54]$. Two $\mathrm{mCi}$ of ${ }^{131} \mathrm{I}-\mathrm{Na}$ (Perkin Elmer, Boston, MA), $10 \mu \mathrm{g}$ of chloramine-T (Sigma) and $5.0 \mu \mathrm{g}$ of the virus were incubated together for $60 \mathrm{~s}$. The radioactively-labeled virus was purified on a column of Sephadex G-10 (Sigma, St. Louis, MO). Human serum albumin $(5 \mu \mathrm{g})$ was labeled by the chloramine-T method with $1 \mathrm{mCi}$ of ${ }^{125} \mathrm{I}-\mathrm{Na}$ (Perkin Elmer) and purified on a G10 column.

\section{Primary cultures of BMECs}

Primary cultures of MBECS and mouse brain pericytes were prepared from 8-week-old CD-1 mice. All experiments were approved by the local (VA St Louis) animal use committee. BMECs were isolated by a modified method of Szabó et al. [55] and Nakagawa et al. [56]. In brief, the cerebral cortices from 8-week-old CD-1 mice were cleaned of meninges and minced. The homogenate was digested with collagenase type II (200 U/mL; Invitrogen, Carlsbad, CA) and DNase I (30 U/mL; Sigma,) in Dulbecco's modified Eagle's medium (DMEM) (Invitrogen) containing 100 units $/ \mathrm{mL}$ penicillin, $100 \mu \mathrm{g} / \mathrm{mL}$ streptomycin, $50 \mu \mathrm{g} / \mathrm{mL}$ gentamicin and $2 \mathrm{mM}$ GlutaMAX ${ }^{\mathrm{m}}-\mathrm{I}$ (Invitrogen) at $37^{\circ} \mathrm{C}$ 
for $40 \mathrm{~min}$. The pellet was separated by centrifugation in $20 \%$ bovine serum albumin $($ BSA)-DMEM $(1,000 \times \mathrm{g}$ for $20 \mathrm{~min})$ to remove neuron and glial cells. The microvessels obtained in the pellet were further digested with collagenase/dispase (1 mg/mL; Roche, Mannheim, Germany) and DNase I (30 U/mL) in DMEM at $37^{\circ} \mathrm{C}$ for $30 \mathrm{~min}$. Microvessel fragments containing pericytes and endothelial cells were separated by a 33\% continuous Percoll (Amersham Biosciences, Piscataway, NJ) gradient centrifugation $(1,000 \times \mathrm{g}$ for $10 \mathrm{~min})$ collected and washed in DMEM $(1,000 \times \mathrm{g}$ for $10 \mathrm{~min})$. To obtain BMECs, the obtained microvessel fragments were seeded on $60 \mathrm{~mm}$ culture dishes (BD FALCON ${ }^{\mathrm{rm}}$, BD Biosciences, Franklin Lakes, NJ) coated with $0.05 \mathrm{mg} / \mathrm{mL}$ fibronectin (Sigma), $0.05 \mathrm{mg} / \mathrm{mL}$ collagen I (Sigma) and $0.1 \mathrm{mg} / \mathrm{mL}$ collagen IV (Sigma). They were incubated in DMEM/Nutrient mixture F-12 HAM (DMEM/F-12) (Invitrogen) supplemented with $20 \%$ plasma derived bovine serum (PDS, Animal Technologies, Tyler, TX), 100 units $/ \mathrm{mL}$ penicillin, $100 \mu \mathrm{g} / \mathrm{mL}$ streptomycin, $50 \mu \mathrm{g} / \mathrm{mL}$ gentamicin, $2 \mathrm{mM}$ GlutaMAX ${ }^{\mathrm{Tm}}-\mathrm{I}$ and $1 \mathrm{ng} / \mathrm{mL}$ basic fibroblast growth factor (bFGF; Sigma) at $37^{\circ} \mathrm{C}$ with a humidified atmosphere of $5 \% \mathrm{CO}_{2} / 95 \%$ air. The next day, the BMECs migrated from the isolated capillaries and started to form a continuous monolayer. To eliminate contaminating cells (mainly pericytes), BMECs were treated with $4 \mu \mathrm{g} / \mathrm{mL}$ puromycin (Sigma) for the first 2 days [57]. After 2 days of the treatment, puromycin was removed from the culture medium. Culture medium was changed every other day. After 7 days in culture, BMECs typically reached $80-90 \%$ confluence.

\section{Primary cultures of pericytes}

Mouse brain pericytes were obtained by a prolonged, 2week culture of isolated brain microvessel fragments under selective culture conditions because brain microvessel fragments contain pericytes [56,58,59]. Brain microvessel fragments were isolated as described above for microvessels, and then the obtained fragments were seeded on uncoated $60 \mathrm{~mm}$ culture dishes (BD FALCON ${ }^{\mathrm{Tm}}$ ) in DMEM containing 20\% fetal bovine serum (FBS), 100 units $/ \mathrm{mL}$ penicillin, $100 \mu \mathrm{g} / \mathrm{mL}$ streptomycin, $50 \mu \mathrm{g} / \mathrm{mL}$ gentamicin, $2 \mathrm{mM}$ GlutaMAX ${ }^{\mathrm{TM}}-\mathrm{I}$ and incubated at $37^{\circ} \mathrm{C}$ with a humidified atmosphere of $5 \% \mathrm{CO}_{2} / 95 \%$ air. The culture medium was changed twice a week. After 14 days in culture, pericytes typically reached $80-90 \%$ confluence. Brain pericytes were characterized by positive immunostaining for a-smooth muscle actin and negative for von Willebrand factor and were used at passage 2-3.

\section{Monoculture and co-culture methods}

Monocultures of BMEC were used to produce conditioned media and to study I-HIV permeability. Monocultures of pericytes were used in cytokine assays, and co-cultures of BMECs with pericytes were used in I-HIV permeability studies (Table 1, Figure 1). BMEC monolayers were obtained by seeding BMECs $\left(4 \times 10^{4}\right.$ cells/ well) on the inside of the fibronectin-collagen IV ( 0.1 and $0.5 \mathrm{mg} / \mathrm{mL}$, respectively)-coated polyester membrane $\left(0.33 \mathrm{~cm}^{2}, 0.4 \mu \mathrm{m}\right.$ pore size $)$ of a Transwell ${ }^{\circledR}$-Clear insert (Costar) and cultured in DMEM/F-12 supplemented with 20\% PDS, 100 units $/ \mathrm{mL}$ penicillin, $100 \mu \mathrm{g} / \mathrm{mL}$ streptomycin, $50 \mu \mathrm{g} / \mathrm{mL}$ gentamicin, $2 \mathrm{mM}$ GlutaMAX ${ }^{\mathrm{m}}-\mathrm{I}, 1 \mathrm{ng} /$ $\mathrm{mL}$ bFGF and $500 \mathrm{nM}$ hydrocortisone (Sigma) at $37^{\circ} \mathrm{C}$ with a humidified atmosphere of $5 \% \mathrm{CO}_{2} / 95 \%$ air until the BMECs reached confluence (3 days). For monocultures of brain pericytes, cells were seeded $\left(4 \times 10^{4}\right.$ cells/ well) into 24-well culture plates (Costar, Corning, NY) and used after a few days.

Co-culture of BMECs and brain pericytes was as described previously [58]; after a few days of pericyte culture as outlined above, BMECs $\left(4 \times 10^{4}\right.$ cells/well $)$ were seeded on the inside of the fibronectin-collagen IV ( 0.1 and $0.5 \mathrm{mg} / \mathrm{mL}$, respectively)-coated polyester membrane $\left(0.33 \mathrm{~cm}^{2}, 0.4 \mathrm{~mm}\right.$ pore size $)$ of a Transwell ${ }^{\circledR}$ Clear insert (Costar) placed in the well of a 24-well culture plate containing layers of brain pericytes (pericyte co-culture) and cultured for 3 days. To check the barrier integrity of BMECs in the BMEC monolayer and pericyte co-culture systems, transendothelial electrical resistance (TEER in $\Omega \times \mathrm{cm}^{2}$ ) was measured before the experiments and after an exposure of LPS using an EVOM voltohmmeter equipped with STX-2 electrode (World Precision Instruments, Sarasota, FL). The TEER of cellfree Transwell ${ }^{\circledR}$-Clear inserts were subtracted from the obtained values.

\section{Measurement of permeability across BMEC}

Lipopolysaccharide from Salmonella typhimurium (LPS; Sigma) was dissolved in serum-free DMEM/F-12 (DMEM/ F-12 containing $1 \mathrm{ng} / \mathrm{ml} \mathrm{bFGF}$ and $500 \mathrm{nM}$ hydrocortisone) to concentrations of 10,50 , and $100 \mu \mathrm{g} / \mathrm{ml}$. To initiate exposure of in vitro BBB models prepared as above to LPS, the culture medium containing serum in both the luminal and abluminal chambers was removed and then serum-free DMEM/F-12 was added to the abluminal chamber. Immediately, medium containing various concentrations of LPS $(10,50,100 \mu \mathrm{g} / \mathrm{ml})$ was added to the

Table 1 Effects of pericytes on transport rates of HIV-1(I-HIV) and albumin (I-Alb) and on transendothelial electrical resistance (TEER) in Monolayers of mouse brain microvascular Endothelial Cells (BMEC)

\begin{tabular}{llll}
\hline & I-HIV & I-Alb & TEER \\
\hline BMEC: no pericytes & $11.5+/-0.9(15)$ & $6.2+/-0.4(15)$ & $60+/-3(15)$ \\
BMEC: + pericytes & $9.0+/-0.4(14)^{*}$ & $6.3+/-0.3(14)$ & $75+/-4(15)^{*}$ \\
\hline
\end{tabular}

Means are reported with their means =/- SE (n). I-HIV and I-Alb transport rates are in $\mathrm{cm} / \mathrm{min}\left(10^{-6}\right)$ and TEER is in $\Omega \times \mathrm{cm}^{2}$. ${ }^{*}$ Statistically different from corresponding "no pericyte" group, $p<0.05$. 


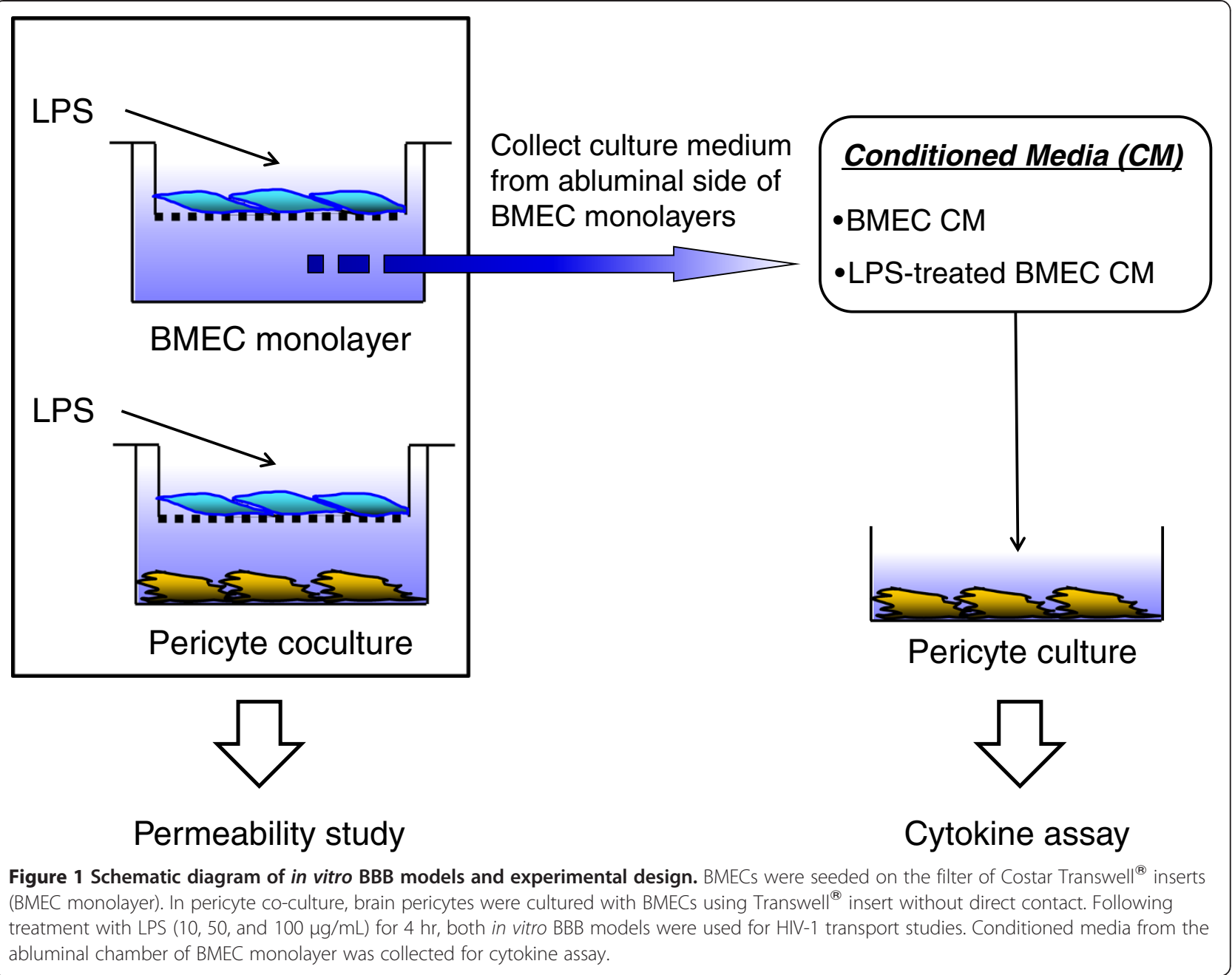

luminal chamber of the co-culture. Serum-free DMEM/F12 without LPS was used as the control medium. TEER was measured at the end of $4 \mathrm{~h}$ incubation at $37^{\circ} \mathrm{C}$. To initiate transport experiments, the medium was removed and BMECs were washed with physiological buffer containing 1\% BSA (141 mM NaCl, $4.0 \mathrm{mM} \mathrm{KCl}, 2.8 \mathrm{mM}$ $\mathrm{CaCl}_{2}, 1.0 \mathrm{mM} \mathrm{MgSO}$, $1.0 \mathrm{mM} \mathrm{NaH} \mathrm{PO}_{4}, 10 \mathrm{mM}$ HEPES, $10 \mathrm{mM}$ D-glucose and 1\% BSA, pH 7.4). The physiological buffer containing $1 \%$ BSA was added to the outside (abluminal chamber; $0.6 \mathrm{~mL}$ ) of the Transwell ${ }^{\circledR}$ insert and ${ }^{131} \mathrm{I}$-HIV-1 $\left(3 \times 10^{6} \mathrm{cpm} / \mathrm{mL}\right)$ was loaded into the luminal chamber. Samples were removed from the abluminal chamber at 15, 30, 60 and 90 min and immediately replaced with an equal volume of fresh $1 \%$ BSA/ physiological buffer. The sampling volume from the abluminal chamber was $0.5 \mathrm{~mL}$. All samples were mixed with 30\% trichloroacetic acid (TCA; final concentration $15 \%)$ and centrifuged at $5,400 \times \mathrm{g}$ for $15 \mathrm{~min}$ at $4^{\circ} \mathrm{C}$. Radioactivity in the TCA precipitate was determined in a gamma counter. The permeability coefficient and clearance of TCA-precipitable ${ }^{131}$ I-HIV-1 was calculated according to the method described by Dehouck et al. [60]. Clearance was expressed as microliters $(\mu \mathrm{L})$ of radioactive tracer diffusing from the luminal to abluminal (influx) chamber and was calculated from the initial level of radioactivity in the loading chamber and final level of radioactivity in the collecting chamber:

$$
\text { Clearance }(\mu \mathrm{L})=[\mathrm{C}]_{\mathrm{C}} \times \mathrm{V}_{\mathrm{C}} /[\mathrm{C}]_{\mathrm{L}} \text {, }
$$

where $[C]_{L}$ is the initial radioactivity in a $\mu \mathrm{L}$ of loading chamber (in $\mathrm{cpm} / \mu \mathrm{L}$ ), $\mathrm{C}_{\mathrm{C}}$ is the radioactivity in a $\mu \mathrm{L}$ of collecting chamber (in $\mathrm{cpm} / \mu \mathrm{L}$ ), and $\mathrm{V}_{\mathrm{C}}$ is the volume of collecting chamber (in $\mu \mathrm{L}$ ). During a 90-min period of the experiment, the clearance volume increased linearly with time. The volume cleared was plotted versus time, and the slope was estimated by linear regression analysis. The slope of clearance curves for the BMEC monolayer plus Transwell ${ }^{\circledR}$ membrane was denoted by $\mathrm{PS}_{\text {app }}$, where PS is the permeability $\times$ surface area product (in $\mu \mathrm{L} / \mathrm{min}$ ). The slope of the clearance curve with a Transwell ${ }^{\circledR}$ membrane without BMECs was denoted by $\mathrm{PS}_{\text {membrane. The real PS }}$ 
value for the BMEC monolayer $\left(\mathrm{PS}_{\mathrm{e}}\right)$ was calculated from $1 / \mathrm{PS}_{\text {app }}=1 / \mathrm{PS}_{\text {membrane }}+1 / \mathrm{PS}_{\mathrm{e}}$. The $\mathrm{PS}_{\mathrm{e}}$ values were divided by the surface area of the Transwell ${ }^{\circledR}$ inserts $\left(0.33 \mathrm{~cm}^{2}\right)$ to generate the endothelial permeability coefficient $\left(\mathrm{P}_{\mathrm{e}}\right.$, in $\left.\mathrm{cm} / \mathrm{min}\right)$.

\section{Cytokine detection}

To obtain BMEC-conditioned media, the abluminal chamber of the BMEC monolayer was filled with $600 \mu \mathrm{L}$ of serum-free DMEM/F-12. At the same time $100 \mu \mathrm{L}$ of serum-free DMEM/F-12 (DMEM/F-12 containing $1 \mathrm{ng} /$ $\mathrm{ml}$ bFGF and $500 \mathrm{nM}$ hydrocortisone) without or with $100 \mu \mathrm{g} / \mathrm{ml}$ LPS was added to the luminal chamber of the BMEC monolayer as described above. After $4 \mathrm{~h}$ of exposure, the fluid in the abluminal chamber of the BMEC monolayer $(600 \mu \mathrm{L})$ was collected as the BMEC conditioned media, sterilized by passing it through a $0.45 \mu \mathrm{m}$ filter syringe, and stored at $-80^{\circ} \mathrm{C}$ until use. Brain pericytes $\left(4 \times 10^{4}\right.$ cells/well $)$ were seeded on the wells of 24-well culture plate (Costar). We divided the pericyte cultures into 4 groups: pericytes treated with (1) BMEC conditioned medium, (2) LPS-treated BMECconditioned medium, (3) serum-free DMEM/F-12 containing no LPS (control), and (4) serum-free DMEM/ F-12 containing LPS $(100 \mu \mathrm{g} / \mathrm{mL})$ where these media are from above. Pericytes were washed with serum-free DMEM/F-12, and then exposed to $200 \mu \mathrm{L}$ of BMECconditioned medium, LPS-treated BMEC conditioned medium, or serum-free DMEM/F-12 with or without LPS $(100 \mu \mathrm{g} / \mathrm{mL})$ for $4 \mathrm{hr}$ at $37^{\circ} \mathrm{C}$. Culture supernatant $(200 \mu \mathrm{L})$ was collected and stored at $-80^{\circ} \mathrm{C}$ until use. The 22 cytokines [granulocyte colony-stimulating factor (G-CSF), granulocyte-macrophage colony-stimulating factor (GM-CSF), interferon gamma (IFN-g), interleukin-1 alpha (IL-1a), IL-1b, IL-2, IL-4, IL-5, IL-6, IL-7, IL-10, IL-12 (p70), IL-13, IL-15, IL-17, interferon-inducible protein-10 (IP-10), keratinocyte chemoattractant (KC), monocyte chemoattractant protein-1 (MCP-1), macrophage inflammatory protein-1 alpha (MIP-1a), regulated upon activation, normal T-cell expressed and secreted (RANTES), and tumor necrosis factor-alpha (TNF-a)] in the collected supernatants, BMEC conditioned medium, and LPStreated BMEC conditioned medium were measured with the mouse cytokine/chemokine Lincoplex ${ }^{\circledR}$ kit (Linco Research, St. Charles, MO) by following the manufacturer's instructions.

\section{Statistical analysis}

Values are expressed as means \pm SEM. One-way and two-way analysis of variances (ANOVAs) followed by Tukey-Kramer's test were applied to multiple comparisons in the permeability study. In the cytokine assay, one-way ANOVA followed by Tukey-Kramer's test were applied to multiple comparisons among the control,
BMEC conditioned medium, and LPS-treated BMEC conditioned medium groups. Student's t-test was applied to comparison between the control and LPS groups. The differences between means were considered to be significant when $P$ values were less than 0.05 using Prism 5.0 (GraphPad, San Diego, CA).

\section{Results}

\section{Pericytes affect permeability}

Table 1 shows that co-culturing with pericytes improved brain endothelial cell monolayer TEER but had no effect on albumin permeability. I-HIV crossed the BMEC monolayers with or without pericyte co-cultures faster than IAlb despite the much larger size of I-HIV. Pericytes reduced the permeation of I-HIV across the BBB to a statistically significant degree $(t=2.60, d f=27, p<0.05)$. These results show that monolayers with or without pericytes are more permeable to HIV-1 than the much smaller albumin, demonstrating that HIV-1 is crossing BMEC by a process other than leakage. The results also show that pericytes have differing effects on the three mechanisms of permeability illustrated in this table: increasing TEER (decreasing paracellular permeability) and decreasing HIV permeability (adsorptive transcytosis), but not affecting albumin permeability (macropinocytosis).

\section{LPS affects permeability}

Addition of LPS produced a dose-dependent increase in IHIV-1 transport across the BMEC monolayer. Results (Figure 2) were expressed as percent of control in order to reduce statistical variance and to allow comparison across treatments and trials. For I-HIV, two-way ANOVA showed significant effects for culture conditions [with or without pericytes: $\mathrm{F}(1,76)=29.5, p<0.001]$ and treatment [LPS concentration: $\mathrm{F}(3,76)=27.5, p<0.001$ ] with no effect for interaction. Tukey's multiple comparison test showed that 50 and $100 \mu \mathrm{g} / \mathrm{ml}$ of LPS increased I-HIV transport across the monolayer in the absence of pericytes and that 10,50 , and $100 \mu \mathrm{g} / \mathrm{ml}$ of LPS increased I-HIV transport across the monolayer in the presence of pericyte coculture. Additionally, any given concentration of LPS produced a statistically greater transport of HIV-1 in the monolayers co-cultured with pericytes when compared to the monocultures (Figure 2 upper panel).

LPS also decreased TEER (Figure 2, lower panel) as shown by the two-way ANOVA that found effects for culture conditions $[\mathrm{F}(1,76)=14.8, p<0.001]$ and LPS treatment $[\mathrm{F}(3,76)=111.5, p<0.001]$. However, Tukey's multiple comparisons test found no effect of pericytes on the LPS-induced decrease in TEER.

\section{Effects of cytokine release}

To further investigate the mechanism by which pericytes, LPS, and brain endothelial cells interact, we 


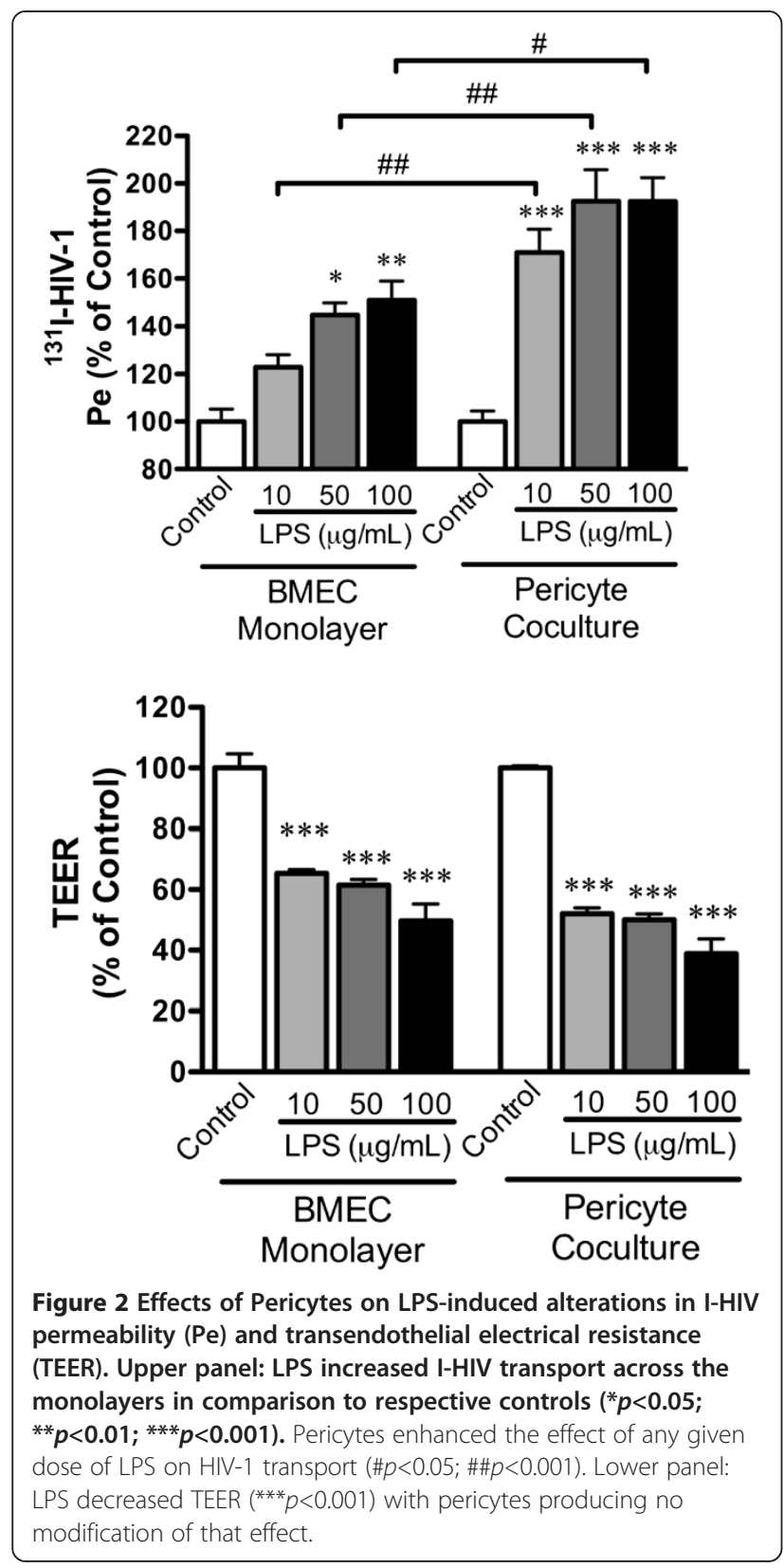

determined whether culture media obtained from brain endothelial cells could induce cytokine release from pericytes. We determined the ability of pericytes to release cytokines either constitutively or when stimulated by LPS. Of 22 cytokines measured, seven (G-CSF, GM-CSF, IL-1 alpha, IL-6, IP-1-, KC, and MCP-1) were constitutively released by pericytes and two others (IL-5 and RANTES) were induced when pericytes were exposed to LPS for $4 \mathrm{~h}$ (Figure 3). LPS also caused a statistically significant increase in the release of each of the seven constitutively- released cytokines, $p<0.01$ (Figure 3).

\section{Endothelial-pericyte crosstalk}

We then incubated pericytes with culture media obtained from brain endothelial cells; in some cases, the endothelial cells had been exposed to LPS. Table 2 shows that four cytokines were detectable in the endothelial cell-conditioned culture media. Of the nine cytokines released by pericytes (Figure 3), endothelial culture media only affected the release of two of them (Figure 4): $\mathrm{KC}[\mathrm{F}(2,14)=58.8$, $p<0.001]$ and MCP-1 $[\mathrm{F}(2,14)=137, p<0.001]$. In both cases, culture media from endothelial cells that had been exposed to LPS, but not culture media from cells that were unexposed, produced the increase.

\section{Discussion}

We first studied the effects of pericytes on the permeability of brain endothelial cell monolayers. The barrier function of brain endothelial cells occurs through a low level of transcytotic mechanisms (macropinocytosis and fenestrae), and an increase in paracellular mechanisms (intercellular tight junctions) [61]. To some degree, the mechanisms that control and alter transcytotic events are different from those controlling paracellular permeability $[62,63]$. Electrical resistance measured by TEER measures channel connections typically ascribed to paracellular permeation. Here, TEER was very low, consistent with little effect on paracellular channel formation. Albumin permeation, however, was also low, indicating

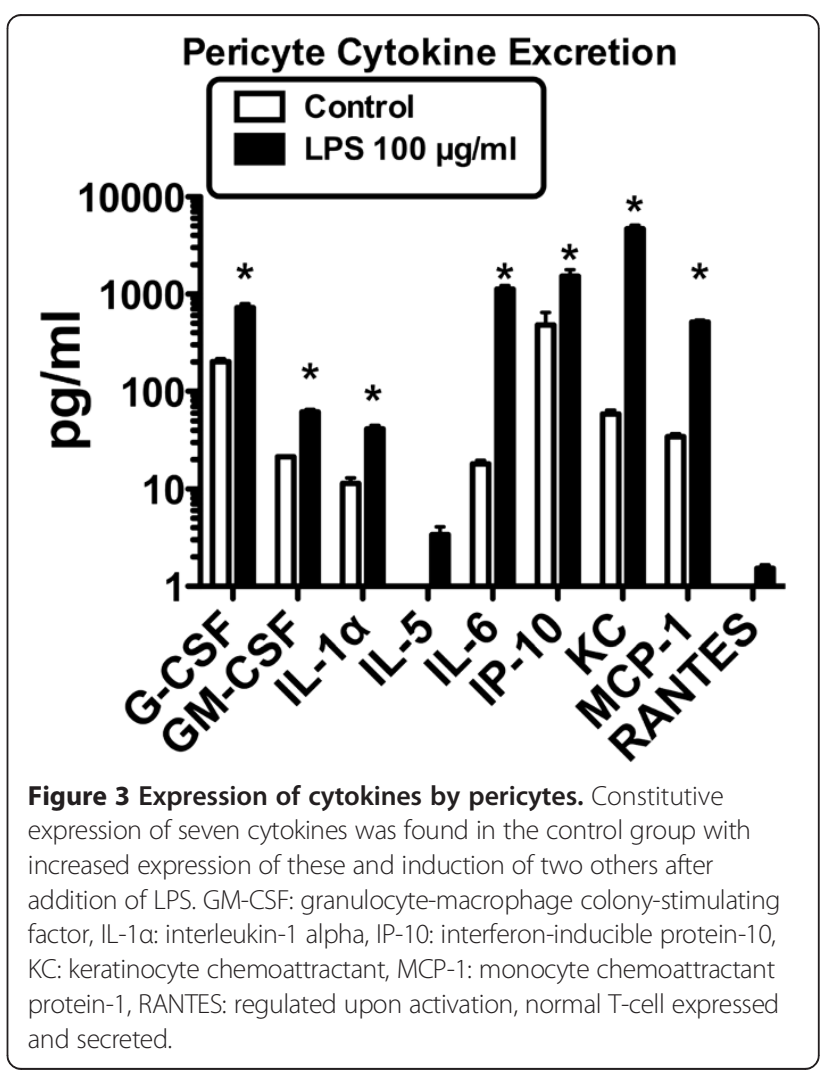


Table 2 Concentrations of cytokines in abluminal culture media from BMECs exposed or not exposed to luminal LPS

\begin{tabular}{lllll}
\hline & G-CSF & IL-1 alpha & IP-10 & KC \\
\hline No LPS & $171+/-6$ & $9.5+/-1.4$ & $18.8+/-2.9$ & 0 \\
LPS & $191+/-9$ & $9.0+/-1.1$ & $19.6+/-5.3$ & $7.8+/-0.9$
\end{tabular}

This culture media was subsequently added to cultures of pericytes (Figure 4). Cytokines as designated in legend to Figure 3. Means +/- SE are shown, $\mathrm{n}=5 /$ cell. Units are $\mathrm{pg} / \mathrm{ml}$.

that transcytotic mechanisms were likely to be low. Mature fenestrae would produce both a low TEER and high albumin permeation, so it is likely that fenestrae were also not present in large numbers in these cells. Coculturing pericytes with the brain endothelial cells significantly improved TEER, suggesting improved tight junction function, but did not alter albumin permeation (Table 1). BMEC-pericyte co-cultures had significantly reduced permeation of I-HIV. Although the HIV-1 virus is much larger than the albumin molecule, the rate at which I-HIV crossed the brain endothelial cell monolayer was much faster than that of I-Alb regardless of whether the endothelial cells were co-cultured with pericytes. This is consistent with the vesicular process for HIV-1 transport being independent of nonspecific vesicular mechanisms such as macropinocytosis.

LPS produced an increase in I-HIV permeation in the monolayers co-cultured and not co-cultured with pericytes. LPS also decreased TEER (that is, it increased paracellular permeability); therefore, this increase in I-HIV could be mediated by transcytotic or paracellular mechanisms. However, the presence of pericytes magnified the LPS effect on HIV-1 permeability without affecting TEER. This suggests that the pericyte-dependent portion of LPS-enhanced IHIV permeation is transcytotic rather than paracellular.

The above results show that pericytes facilitate the LPSenhanced transport of HIV-1 across brain endothelial cells. We then investigated some of the mechanisms by which pericytes could mediate such facilitation. LPS crosses the in vivo BBB, even the disrupted BBB, poorly if at all [64]. In vitro, LPS produces different patterns of cytokine release when added to the luminal versus the abluminal chamber, consistent with an inability to cross the in vitro $\mathrm{BBB}$ as well $[37,65]$. We, therefore, postulated that LPS was likely acting at the luminal surface of the brain endothelial cell, rather than at the pericytes in the abluminal chamber, as the first step in a neuroimmunebased modulation of the crosstalk between pericytes and brain endothelial cells. More specifically, we postulated that LPS acts at the luminal surface of the brain endothelial cell to induce release of soluble factors from its abluminal surface. These soluble factors would then act on pericytes, inducing them to release soluble factors that would modulate brain endothelial cell transcytosis of IHIV. To test this hypothesis, we examined the release of

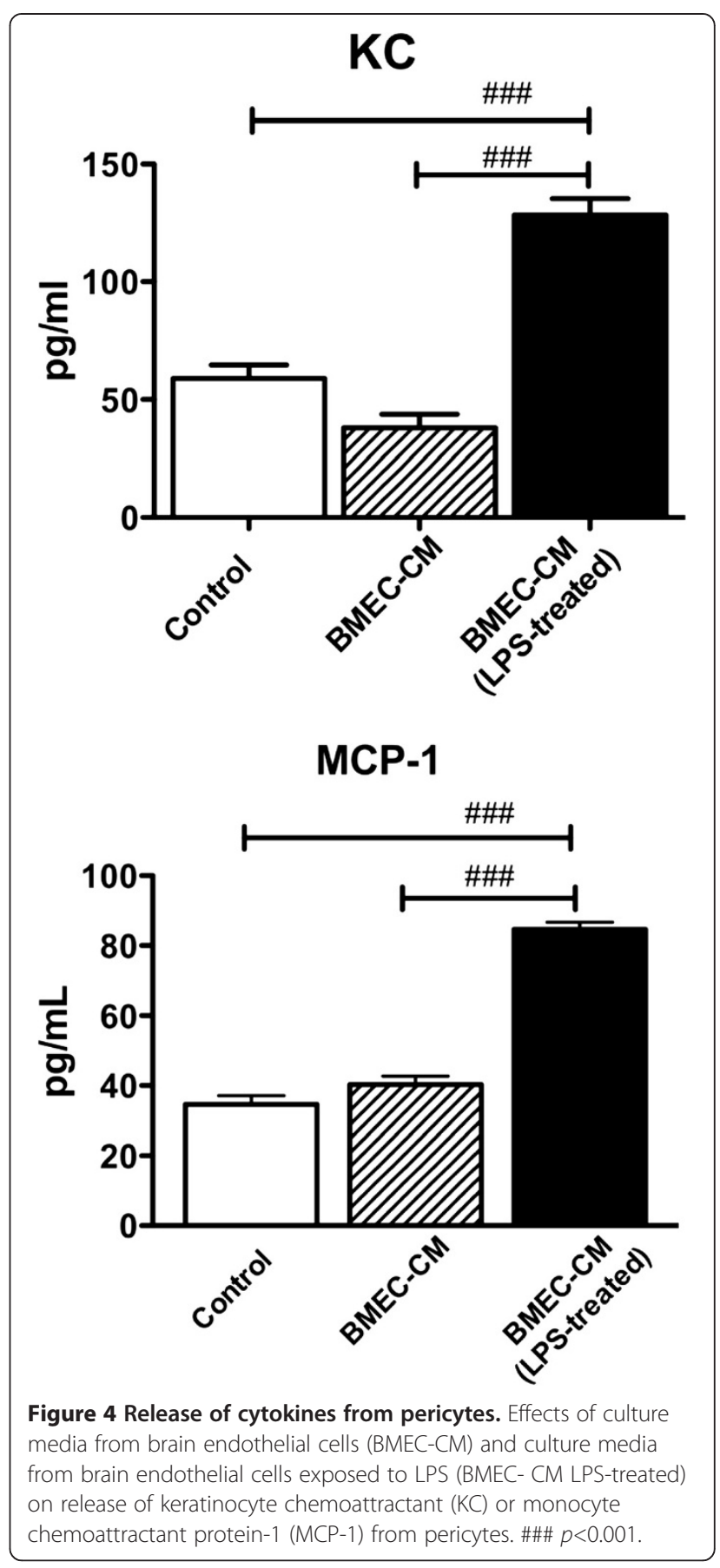

cytokines from BMECs and pericytes. It is known that both endothelial cells and pericytes release cytokines, that cytokines are important in communication between the cells of the neurovascular unit, that cytokines enhance HIV-1 transcytosis, and that LPS can act on one side of the $\mathrm{BBB}$ to affect the release of cytokines from the other side $[37,44,66,67]$.

We first exposed the luminal surface of BMEC monolayers to a short, $4 \mathrm{~h}$ exposure to LPS, then collected the abluminal culture media from these monolayers and 
abluminal culture media from BMEC monolayers not exposed to LPS was used as a control. We then assessed the ability of the culture media to release cytokines from pericytes. We found that culture media from LPSexposed brain endothelial cells did indeed increase the release of two cytokines, KC and MCP-1, from pericytes. The level of these cytokines was much higher in the pericyte culture medias (40-140 $\mathrm{pg} / \mathrm{ml}$; see Figure 4) than in the brain endothelial cell culture medias (see Table 2), thus ruling out contamination as a source of these results. Likewise, it is unlikely that LPS from the endothelial cell culture media caused this stimulation as the LPS was added to the luminal chamber of the endothelial cells, whereas the media exposed to the pericytes was from the abluminal chamber. Furthermore, LPS contamination would have been expected to produce the cytokine profile observed in Figure 3 and not just increases in KC and MCP-1. Therefore, the most parsimonious explanation of our results fits our hypothetical model: LPS acting at the luminal surface of brain endothelial cells stimulates release of soluble factors from their abluminal surface which then modulates pericyte release of immune-active factors.

The levels of release of KC and MCP-1 are substantial. Given that the pericyte cultures contained about $50 \mu \mathrm{g}$ of protein, we estimate $\mathrm{MCP}-1$ and $\mathrm{KC}$ concentrations of 960 and $1440 \mathrm{pg} / \mathrm{mg}$ protein. This exceeds the levels produced in brain after in vivo administration of LPS [67].

Although we found the release of two cytokines from pericytes was specifically affected, it is likely that the release of many other immune active substances is similarly modulated. However, pericyte-secreted MCP-1 could be directly involved in the enhanced transcytosis of HIV-1. MCP-1 derived from astrocytes [68] and microglia [69] mediates cross talk with brain endothelial cells that increases the diapedesis across the BBB of HIV-1 infected macrophages and monocytes. MCP-1 also affects voltagegated potassium channels in brain endothelial cells [70]. Here, the evidence shows that pericytes are also a source of MCP-1 which is released in response to signals from brain endothelial cells. It could be that MCP-1 released from pericytes alters brain endothelial transcytotic processes that affect the permeation of free HIV-1.

\section{Conclusion}

Pericytes potentiate the LPS-enhanced transport of HIV-1 across BMECs. This pericyte potentiation does not affect TEER and so is likely mediated through transcytotic mechanisms. We conclude that LPS acts at the luminal surface of the BMEC monolayer, inducing abluminal secretion of cytokines and other substances that in turn act on pericytes, the pericytes then secrete factors that enhance I-HIV transcytosis across the BMECs.

\section{Competing interests}

The authors do not have financial or nonfinancial competing interests.

\section{Authors' contributions}

Both WAB and SD contributed to experimental design, statistical analysis, and writing of the MS. Experimental work was carried out by SD. All authors read and approved the final manuscript.

\section{Acknowledgments}

Supported by VA merit review and R0-1 DK083485.

Received: 16 November 2012 Accepted: 23 June 2013

Published: 1 July 2013

\section{References}

1. Groothuis DR, Levy RM: The entry of antiviral and antiretrovial drugs into the central nervous system. J Neurovirol 1997, 3:387-400.

2. Kim RB, Fromm MF, Wandel C, Leake B, Wood AJJ, Roden DM, Wilkinson GR: The drug transporter $\mathrm{p}$-glycoprotein limits oral absorption and brain entry of HIV-1 protease inhibitors. J Clin Invest 1998, 101:289-294.

3. Thomas SA: Anti-HIV drug distribution to the central nervous system. Curr Pharmaceut Des 2004, 10:1313-1324.

4. Bansal AK, Mactutus CF, Nath A, Maragos W, Hauser KF, Booze RM: Neurotoxicity of HIV-1 proteins gp120 and Tat in the rat striatum. Brain Res 2000, 879:42-49.

5. Benos DJ, McPherson S, Hahn BH, Chaikin MA, Benveniste EN: Cytokines and HIV envelope glycoprotein gp120 stimulate $\mathrm{Na}+/ \mathrm{H}+$ exchange in astrocytes. J Biol Chem 1994, 269:13811-13816.

6. Bottner A, Mehraein P, Weis S: Vascular changes in the cerebral cortex in HIV-1 infection. Acta Neuropathol 1996, 92:35-41.

7. Farr SA, Banks WA, Uezu K, Freed EO, Kumar VB, Morley JE: Mechanisms of HIV-1 induced cognitive impairment: evidence for hippocampal cholinergic involvement with overstimulation of the VIPergic system by the viral coat protein core. AIDS Res Hum Retroviruses 2002, 18:1189-1195.

8. Fujimura RK, Bockstahler LE, Goodkin K, Werner T, Brack-Werner R, Shapshak P: Neuropathology and virology of HIV associated dementia. Med Virol 1996, 6:141-150.

9. Gendelman HE, Zheng J, Coulter CL, Ghorpade A, Che M, Thylin M, Rubocki R, Persidsky Y, Hahn F, Reinhard J Jr, Swindells S: Suppression of inflammatory neurotoxins by highly active antiretroviral therapy in human immunodeficiency virus-associated dementia. J Infect Dis 1998, 178:1000-1007.

10. Grossman DM, Banks WA, LeBlanc J, Dejace P: Prevalence of hypernatremia in HIV-infected VA patients. J Invest Med 1998, 46:46A.

11. Opp MR, Hughes TK Jr, Smith EM: HIV-1 glycoprotein 120 alters rat sleep. The Physiologist 1994, 37(3):A-50.

12. Bagasra O, Lavi E, Bobroski L, Khalili K, Pestaner JP, Tawadros R, Pomerantz RJ: Cellular reservoirs of HIV-1 in the central nervous system of infected individuals: indentification by the combination of in situ polymerase chain reaction and immunohistochemistry. AIDS 1996, 10:573-585.

13. Nottet HS, Persidsky Y, Sasseville VG, Nukuna AN, Bock P, Zhai QH, Sharer LR, McComb RD, Swindells S, Soderland C, Gendelman HE: Mechanisms for the transendothelial migration of HIV-1-infected monocytes into brain. J Immunol 1996, 156:1284-1295.

14. Persidsky Y, Stins M, Way D, Witte MH, Weinand M, Kim KS, Bock P, Gendelman HE, Fiala M: A model for monocyte migration through the blood-brain barrier during HIV-1 encephalitis. J Immunol 1997, 158:3499-3510.

15. Banks WA, Freed EO, Wolf KM, Robinson SM, Franko M, Kumar VB: Transport of human immunodeficiency virus type 1 pseudoviruses across the blood-brain barrier: role of envelope proteins and adsorptive endocytosis. J Virol 2001, 75:4681-4691.

16. Anathbandhu C, Yang B, Gendelman HE, Persidsky Y, Kanmogne GD: STAT1 signaling modulates HIV-1-induced inflammatory responses and leukocyte transmigration across the blood-brain barrier. Blood 2007 111:2062-2072.

17. Ramirez SH, Fan S, Dykstra H, Reichenbach N, Del Valle L, Potula R, Phipps RP, Maggirwar SB, Persidsky Y: Dyad of CD40/CD40 ligand fosters neuroinflammation at the blood-brain barrier and is regulated via JNK signaling: implications for HIV-1 encephalitis. J Neurosci 2010, 30:9454-9464 
18. Banks WA, Akerstrom V, Kastin AJ: Adsorptive endocytosis mediates the passage of HIV-1 across the blood-brain barrier: evidence for a post-internalization coreceptor. J Cell Sci 1998, 111:533-540

19. Banks WA, Robinson SM, Wolf KM, Bess JW Jr, Arthur LO: Binding, internalization, and membrane incorporation of human immunodeficiency virus- 1 at the blood-brain barrier is differentially regulated. Neurosci 2004, 128:143-153.

20. Dohgu S, Banks WA: Lipopolysaccharide-enhanced transcellular transport of HIV-1 across the blood-brain barrier is medited by the p38 mitogen-activated protein kinase pathway. Exp Neurol 2008, 210:740-749.

21. Nakaoke R, Ryerse JS, Niwa M, Banks WA: Human immunodeficiency virus type 1 transport across the in vitro mouse brain endothelial cell monolayer. Exp Neurol 2005, 193:101-109.

22. Dohgu S, Ryerse JS, Robinson SM, Banks WA: Human immunodeficiency virus-1 uses the mannos-6-phosphate receptor to cross the blood-brain barrier. PLOS one 2012, 7:e41623.

23. Alonso K, Pontiggia P, Medenica R, Rizzo R: Cytokine patterns in adults with AIDS. Immunol Invest 1997, 26:341-350.

24. Chaudhuri A, Duan F, Morsey B, Persidsky Y, Kanmogne GD: HIV-1 activates proinflammatory and interferon-inducible genes in human brain microvascular endothelial cells: putative mechanisms of blood-brain barrier dysfunction. J Cereb Blood Flow Metab 2007, 28:697-711.

25. Didier N, Banks WA, Creminon C, Dereuddre-Bosquet N, Mabondzo A: HIV-1-induced production of endothelin-1 in an in vitro model of the human blood-brain barrier. Neuroreport 2002, 13:1179-1183.

26. Ulmer AJ, Rietschel ET, Zahringer U, Heine H: Lipopolysaccharide: structure, bioactivity, receptors, and signal transduction. Trends Glycosci Glycotechnol 2002, 14:53-68.

27. Brenchley JM, Price DA, Schacker TW, Asher TE, Silvestri G, Rao S, Kazzaz Z, Bornstein E, Lambotte $O$, Altmann D, et al: Microbial translocation is a cause of systemic immune activation in chronic HIV injection. Nature Med 2006, 12:1365-1371.

28. Laye S, Parnet P, Goujon E, Dantzer R: Peripheral administration of lipopolysaccharide induces the expression of cytokine transcripts in the brain and pituitary of mice. Brain Res 1994, 27:157-162.

29. Banks WA: Physiology and pathophysiology of the blood-brain barrier: Implications for microbial pathogenesis, drug delivery and neurodegenerative disorders. J Neurovirol 1999, 5:538-555.

30. Banks WA, Dohgu S, Nakaoke R, Lynch JL, Fleegal-DeMotta MA Erickson MA, Vo TQ: Nitric oxide isoenzymes regulate LPS-enhanced insulin transport across the blood-brain barrier. Endocrinol 2008, 149:1514-1523.

31. Nonaka N, Shioda S, Banks WA: Effect of lipopolysaccharide on the transport of pituitary adenylate cyclase activating polypeptide across the blood-brain barrier. Exp Neurol 2005, 191:137-144.

32. Xaio H, Banks WA, Niehoff ML, Morley JE: Effect of LPS on the permeability of the blood-brain barrier to insulin. Brain Res 2001, 896:36-42.

33. Hartz AMS, Bauer B, Fricker G, Miller DS: Rapid modulation of Pglycoprotein-mediated transport at the blood-brain barrier by tumor necrosis factor-alpha and lipopolysaccharide. Molec Pharmacol 2006, 69:462-470.

34. Jaeger JB, Dohgu S, Lynch JL, Fleegal-DeMotta MA, Banks WA: Effects of lipopolysaccharide on the blood-brain barrier transport of amyloid beta protein: a mechanism for inflammation in the progression of Alzheimer's disease. Brain Behav, Immunity 2009, 23:507-517.

35. Salkeni MA, Lynch JL, Price TO, Banks WA: Lipopolysaccharide impairs blood-brain barrier P-glycoprotein function in mice through prostaglandin- and nitric oxide-independent pathways and nitric oxide-independent pathways. J Neuroimmune Pharmacology. J Neuroimmune Pharmacol 2009, 4:276-282.

36. Reyes TM, Fabry Z, Coe CL: Brain endothelial cell production of a neuroprotective cytokine, interleukin-6, in response to noxious stimuli. Brain Res 1999, 851:215-220.

37. Verma S, Nakaoke R, Dohgu S, Banks WA: Release of cytokines by brain endothelial cells: a polarized response to lipopolysaccharide. Brain Behav, Immunity 2006, 20:449-455.

38. Andras IE, Pu H, Deli MA, Nath A, Hennig B, Toborek M: HIV-1 Tat protein alters tight junction protein expression and distribution in cultured brain endothelial cells. J Neurosci Res 2003, 74:255-265.

39. Annunziata P: Blood-brain barrier changes during invasion of the central nervous system. J Neurol 2003, 250:901-906.
40. Kanmogne GD, Primeaux C, Grammas P: HIV-1 gp120 proteins alter tight junction protein expression and brain endothelial cell permability: implications for the pathogenesis of HIV-associated dementia. J Neuropathol Exper Neurol 2005, 64:498-505.

41. Hayashi K, Pu H, Andras IE, Eum SY, Yamauchi A, Henning B, Toborek M: HIV-TAT protein upregulates expressin of multidrug resistance protein 1 in the blood-brain barrier. J Cereb Blood Flow and Metab 2006, 26:1052-1065.

42. Persidsky Y, Zheng J, Miller D, Gendelman HE: Mononuclear phagocytes mediate blood-brain barrier compromise and neuronal injury during HIV-1-associated dementia. J Leukocyte Biol 2000, 68:413-422.

43. Toborek M, Lee YW, Flora G, Pu H, Andreeff M, Wylegala E, Henning B, Nath A: Mechanisms of the blood-brain barrier disruption in HIV-1 infection. Cell Molec Neurobiol 2005, 25:181-199.

44. Neuwelt E, Abbott NJ, Abrey L, Banks WA, Blakley B, Davis T, Engelhardt B, Grammas P, Nedergaard M, Nutt J, et al: Strategies to advance translational research into brain barriers. Lancet Neurol 2008, 7:84-96.

45. Katyshev V, Dore-Duffy P: Pericyte coculture models to study astrocyte, pericyte, and endothelial cell interactions. Methods Mol Biol 2012, 814:467-481.

46. Dore-Duffy P: Pericytes: pluripotent cells of the blood brain barrier. Current Pharmaceut Des 2008, 14:1581-1593.

47. Dore-Duffy P, Katychev A, Wang X, Van Buren E: CNS microvascular pericytes exhibit multipotential stem cell activity. J Cereb Blood Flow Metab 2006, 26:613-624.

48. Dore-Duffy P, Owen C, Balabanov R, Murphy S, Beaumont T, Rafols JA Pericyte migration from the vascular wall in response to traumatic brain injury. Microvasc Res 2000, 60:55-69.

49. Bronkowski D, Katyshev V, Balabanov RD, Borisov A, Dore-Duffy P: The CNS microvascular pericyte: pericyte-astrocyte crosstalk in the regulation of tissue survival. Fluids Barriers CNS 2011, 8:8.

50. Nakagawa S, Castro V, Toborek M: Infection of human pericytes by HIV-1 disrupts the integrity of the blood-brain barrier. J Cell Mol Med 2012, 16:2950-2957

51. Arthur LO, Bess JW Jr, Chertova EN, Rossio JL, Esser MT, Benveniste RE, Henderson LE, Lifson JD: Chemical inactivation of retroviral infectivity by targeting nucleocapsid protein zinc fingers: a candidate SIV vaccine. AIDS Res Human Retroviruses 1998, 14(supplement 3):S-311.

52. Rossio JL, Esser MT, Suryanarayana K, Schneider DK, Bess JW Jr, Vasquez GM, Wiltrout TA, Chertova E, Grimes MK, Sattentau Q, et al: Inactivation of human immunodeficiency virus type 1 infectivity with preservation of conformational and functional integrity of virion surface proteins. J Virol 1998, 72:7992-8001.

53. Frost EH: Radioactive labelling of viruses: an iodination preserving biological properties. JGenVirol 1977, 35:181-185.

54. Montelaro RC, Rueckert RR: On the use of chloramine-T to iodinate specifically the surface proteins of intact enveloped viruses. J GenVirol 1975, 29:127-131.

55. Szabo CA, Deli MA, Ngo TKD, Joo F: Production of pure primary rat cerebral endothelial cell culture: a comparison of different methods. Neurobiol 1997, 5:1-16.

56. Nakagawa S, Deli MA, Nakao S, Honda M, Hayashi K, Nakaoke R, Kataoka Y, Niwa M: Pericytes from brain microvessels strengthen the barrier integrity in primary cultures of rat brain endothelial cells. Cell $\mathrm{Mol}$ Neurobiol 2007, 27:687-694.

57. Perriere N, Demeuse P, Garcia E, Regina A, Debray M, Andreux JP, Couvreur P, Scherrmann JM, Temsamani J, Couraud PO, et al: Puromycin-based purification of rat brain capillary endothelial cell cultures. $J$ Neurochem 2005, 93:279-289.

58. Dohgu S, Takata F, Yamauchi A, Nakagawa S, Egawa T, Naito M, Tsuruo T Sawada Y, Niwa M, Kataoka Y: Brain pericytes contribute to the upregulation and maintenance of blood-brain barrier functions through transforming growth factor-beta production. Brain Res 2005, 1038:208-215.

59. Hiyashi K, Nakao S, Nakaoke R, Nakagawa S, Kitagawa K, Niwa M: Effects of hypoxia on endothelial/pericyte co-culture model of the blood-brain barrier. Reg Peptides 2004, 123:77-83.

60. Dehouck MP, Jolliet-Riant P, Bree F, Fruchart JC, Cecchelli R, Tillement JP: Drug transfer across the blood-brain barrier: correlation between in vitro and in vivo models. J Neurochem 1992, 58:1790-1797. 
61. Davson H: The blood-brain barrier. In Physiology of the Cerebrospinal Fluid. London: J. and A. Churchill, LTD; 1967:82-103.

62. Fleegal-DeMotta MA, Dohgu S, Banks WA: Angiotensin II modulates BBB permeability via activation of the AT1 receptor in brain endothelial cells. J Cereb Blood Flow Metab 2009, 29:640-647.

63. Mayhan WG, Heistad DD: Permeability of blood-brain barrier to various sized molecules. Am J Physiology 1985, 248:H712-H718.

64. Banks WA, Robinson SM: Minimal penetration of lipopolysaccharide across the murine blood-brain barrier. Brain, Behav, and Immunity 2010, 2010:102-109

65. Dohgu S, Fleegal-DeMotta MA, Banks WA: Lipopolysaccharide-enhanced transcellular transport of HIV-1 across the blood-brain barrier is mediated by luminal microvessel IL-6 and GM-CSF. J Neuroinflam 2011, 8:167.

66. Kovac A, Erickson MA, Banks WA: Brain microvascular pericytes are immunoactive in culture: cytokine, chemokine, nitric oxide, and LRP-1 expression in response to lipopolysaccharide. J Neuroinflam 2011, 8:139

67. Erickson MA, Banks WA: Cytokine and chemokine responses in serum and brain after single and repeated injections of lipopolysaccharide: mutliplex quantification with path analysis. Brain, Behav, and Immunity 2011, 25:1637-1648.

68. Muratori C, Mangino G, Affabris E, Federico M: Astrocytes contacting HIV-1-infected macrophages increase the release of CCL2 in response to the HIV-1-dependent enhancement of membrane-associated TNF alpha in macrophages. Glia 2010, 58:1893-1904.

69. Yao H, Yang Y, Kim KJ, Bethel-Brown C, Gong N, Funa K, Gendelman HE, Su TP, Wang JQ, Buch S: Molecular mechanisms involving sigma receptor-mediated induction of MCP-1: implications for increased monocyte transmigration. Blood 2010, 115:4951-4962.

70. Gendelman HE, Ding S, Gong N, Liu J, Ramirez SH, Persidsky Y, Mosley RL, Wang T, Volsky DJ, Xiong H: Monocyte chemotactic protein-1 regulates voltage-gated $\mathrm{K}+$ channels and macrophage transmigration. J Neuroimmune Pharmacol 2009, 4:47-59.

\section{doi:10.1186/2045-8118-10-23}

Cite this article as: Dohgu and Banks: Brain pericytes increase the lipopolysaccharide-enhanced transcytosis of HIV-1 free virus across the in vitro blood-brain barrier: evidence for cytokine-mediated pericyte-endothelial cell crosstalk. Fluids and Barriers of the CNS 2013 10:23.

\section{Submit your next manuscript to BioMed Central and take full advantage of:}

- Convenient online submission

- Thorough peer review

- No space constraints or color figure charges

- Immediate publication on acceptance

- Inclusion in PubMed, CAS, Scopus and Google Scholar

- Research which is freely available for redistribution 